\section{Bronchial obstruction due to respiratory mucosal sloughing in toxic epidermal necrolysis}

\author{
A Dasgupta, J O'Malley, R Mallya, \\ $\mathrm{J}$ G Williams
}

\begin{abstract}
A 17 year old woman is described who nearly asphyxiated because of sloughing of the bronchial mucosa as a result of toxic epidermal necrolysis. Bronchoscopic aspiration of bronchial slough proved life saving.
\end{abstract}

(Thorax 1994;49:935-936)

Toxic epidermal necrolysis is an uncommon but severe form of epidermal sloughing. It carries a high mortality rate, usually due to fulminating infection of the skin leading to shock and multiorgan failure. We describe a patient who exhibited bronchial obstruction as a result of severe respiratory mucosal sloughing whilst suffering from the disorder.

\section{Case history}

A 17 year old unemployed woman was admitted with a four day history of increasing headaches, fever, and generalised erythematous rash. Blisters had begun to develop on her face and orocutaneous junction one day before admission. She had only taken paracetamol tablets, and was not receiving any drug treatment before her illness. In particular, she was not taking the oral contraceptive pill.

On examination her temperature was $39^{\circ} \mathrm{C}$ and flaccid bullae with large erythematous sheets affecting the head and neck more than the lower limbs were present. There was sloughing of the oral and genital mucous membranes involving $50 \%$ of the skin surface. There was no evidence of a retained tampon.

She was initially diagnosed as having Stevens-Johnson syndrome and was treated with prednisolone, $40 \mathrm{mg}$ once daily. In view of the lack of response within 48 hours a dermatological opinion was sought, and the diagnosis was revised to toxic epidermal necrolysis. Skin biopsy confirmed the diagnosis. Systemic steroids were discontinued and Hydrous Ointment (BP) was applied to intact skin twice daily and potassium permanganate solution to sloughed areas four times a day. Ciproxin was prescribed as a prophylactic antibiotic. Ocular toilet was performed regularly.

Over the next two days it became apparent that the bronchial mucosa was also sloughing as the patient developed a productive cough with slough associated with slight haemoptysis. Fine basal crepitations were heard at the right lung base. The chest radiograph was normal. Four days after admission she had two acute choking attacks associated with cyanosis. She was then intubated to ensure a patent airway and put on positive pressure ventilation. The pressure required to ventilate her remained high ( $\left.40-50 \mathrm{~cm} \mathrm{H}_{2} \mathrm{O}\right)$. In view of this, fibreoptic bronchoscopy was performed through an endotracheal tube. Slough was seen to be obstructing both main bronchi and was removed on two separate occasions with a wide aspirating fibreoptic bronchoscope. The appearance of the slough was like a bronchial cast (figure). The bronchial orifices appeared to be swollen and erythematous. Subsequent microbiological culture showed no evidence of infection.

Once the respiratory slough was removed the pressure required to ventilate the patient decreased dramatically to $30 \mathrm{~cm} \mathrm{H}_{2} \mathrm{O}$. Her subsequent recovery was delayed by Staphylococcus aureus septicaemia. She was ventilated for a total of 10 days and discharged from hospital three weeks after admission. She lost all her nails which subsequently regrew. Despite ocular toilet with frequent washes she developed cicatrising conjunctivitis and is still under specialised ophthalmological care.

\section{Discussion}

Toxic epidermal necrolysis is a rare condition in which most of the skin of the body becomes erythematous, inflamed, then necrotic following a bullous phase. Non-steroidal antiinflammatory drugs, sulphonamides, and lymphomas are known causes of the condition, but it may also be idiopathic. Patients have a

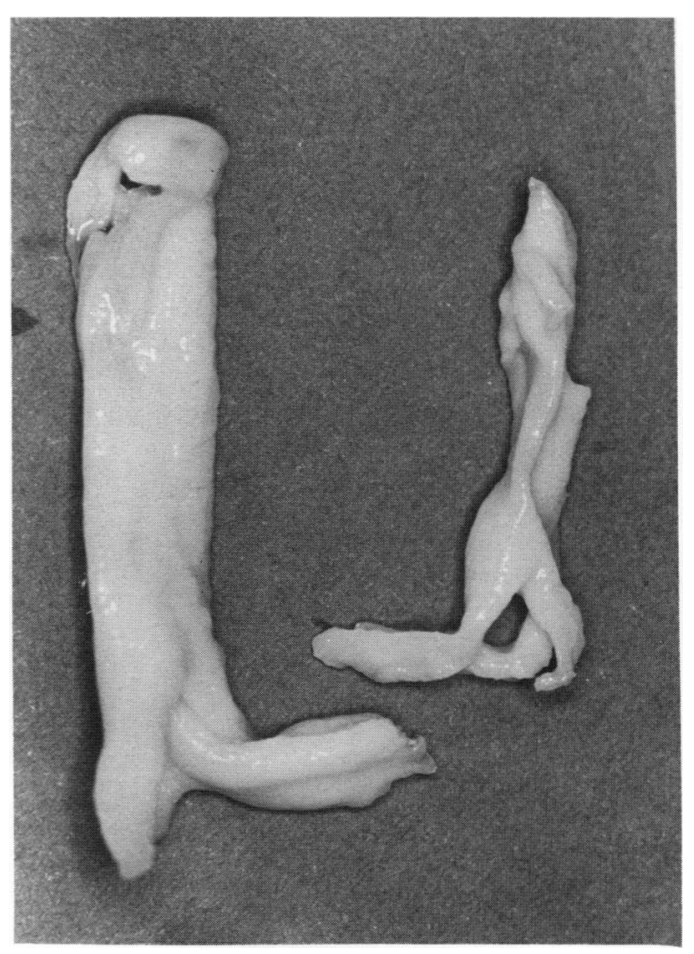

Bronchial cast aspirated at bronchoscopy. 
similar appearance to those afflicted by the staphylococcal scalded skin syndrome, but the level of epidermal cleavage is much deeper in toxic epidermal necrolysis. It is a highly lethal disease with a mortality of $20-70 \%$, even in the best centres. It has a substantial morbidity which includes corneal scarring, contractures, and oesophageal strictures. Pneumonia is a common complication of this disorder. ${ }^{1}$

The plan of management is to keep the affected areas free from infection with applications of local antiseptics. Good nursing is of paramount importance. Systemic steroids are not of proven benefit and may increase the risk of secondary infection. Careful fluid and electrolyte monitoring is required.

Our patient developed symptoms and signs of respiratory mucosal sloughing for 48 hours before her acute choking attacks. The bronchial obstruction was removed with a fibreoptic bronchoscope although this took up to an hour to do on two separate occasions. In the light of this experience we feel that it may be safer to use a rigid bronchoscope at the onset of bronchial mucosal sloughing to facilitate suction of secretions and slough. Tracheostomy may need to be performed to secure the airway patency despite the risks of local sepsis.

This case highlights the existence of a lifethreatening complication of this disorder, and should enable clinicians to plan management of such cases on the basis of our experience.

1 Heimback DM, Engrav LH, Marvin JA, Harnar TJ, Grube BJ. Toxic epidermal necrolysis - a step forward in treatment. fAM $A$ 1987;257:2171-5.

\title{
Pneumothorax following thoracic radiation therapy for Hodgkin's disease
}

\author{
M G Penniment, P C O’Brien
}

\begin{abstract}
Radiation therapy alone to the nodal drainage sites above the diaphragm, namely a "mantle" field, is often standard treatment for early stage Hodgkin's disease and may be used in combination with chemotherapy in more advanced disease. Localised pneumonitis and fibrosis are recognised treatment related sequelae; however, other pulmonary complications, including pneumothorax, have been described. Two cases of spontaneous pneumothorax following mantle radiation therapy are presented.
\end{abstract}

(Thorax 1994;49:936-937)

Department of Radiation Oncology, Royal Adelaide Hospital, Adelaide, South Australia $\mathbf{5 0 0 0}$ M G Penniment PC O'Brien

Reprint requests to: Dr M Penniment.

Received 22 July 1993 Returned to authors 15 October 1993 Revised version received 6 December 1993 Accepted for publication 13 December 1993

\section{Case 1}

An 18 year old non-smoking male university student of medium build presented with left axillary and supraclavicular masses and was subsequently diagnosed as having clinical stage IIA nodular sclerosing Hodgkin's disease. Subtotal nodal irradiation was given, beginning with a mantle field delivering $36 \mathrm{~Gy}$ in 20 fractions, followed by an infradiaphragmatic field delivering the same dose to the para-aortic nodes and spleen. The treatment course was uneventful but five months after completing the chest irradiation he was found to have a right apical pneumothorax on routine chest radiography.

As the patient was asymptomatic and the pneumothorax comprised less than $20 \%$ of the right lung volume, he was observed. Serial chest radiographs showed gradual resolution over a further five months. He continued normal activity, including sports, and has had no evidence of Hodgkin's disease one year after treatment.

\section{Case 2}

A 28 year old non-smoking woman of normal build presented with a four week history of a mass in the left side of her neck and night sweats twice a week for two months. Nodular sclerosing Hodgkin's disease was diagnosed after nodal biopsy, and computed tomographic scanning of the neck and thorax showed a mediastinal mass which was continuous with the right neck disease. She was designated pathological stage IIB following a normal staging laparotomy and proceeded to mantle irradiation to a total dose of $36 \mathrm{~Gy}$.

Follow up chest radiographs indicated that the mediastinal mass had completely responded but five months after treatment bilateral apical pleural capping was noted. The apical changes progressed until a chest radiograph 17 months after treatment showed a small left pneumothorax which had then resolved three months later. Again the patient remained asymptomatic.

\section{Discussion}

The two cases reported demonstrate the complication of pneumothorax occurring after mantle radiation therapy alone for Hodgkin's disease. Both the cases received $36 \mathrm{~Gy}$ and this is consistent with other reported cases who also received more than $30 \mathrm{~Gy}{ }^{1-4}$ There have been too few reported cases to postulate a doseresponse relation. 